\title{
Zur Lage der Genossenschaften - tatsächliche Renaissance oder Wunschdenken?
}

Im UN-Jahr der Genossenschaften gewinnt die Diskussion über genossenschaftliche Organisationsformen wieder an Konjunktur. Die Vielzahl neu gegründeter Genossenschaften jenseits der traditionellen Geschäftsfelder wie der Wohnungswirtschaft und dem Finanzsektor zeigt, dass die Rechtsform der eingetragenen Genossenschaft im 21. Jahrhundert angekommen ist. Offen scheint derweil, wo die neuen Genossenschaften, die häufig in der Energiewirtschaft entstanden sind, innerhalb des wissenschaftlichen Diskurses über Alternativformen des kapitalistischen Wirtschaftens einzuordnen sind.

HERBERT KLEMISCH, MORITZ BODDENBERG

\section{Einleitung und Forschungsfragen}

„Cooperatives are a reminder to the international community that it is possible to pursue both economic viability and social responsibility." Mit diesen Worten begründete UNOGeneralsekretär Ban-Ki Moon die Entscheidung der Vereinten Nationen, das Jahr 2012 zum „Internationalen Jahr der Genossenschaften“ zu erklären, das von Genossenschaftsverbänden, politischen Stiftungen und genossenschaftlichen Forschungsinstitutionen durch Veranstaltungen, Publikationen und Initiativen begleitet wird. Die Diskussion über die konzeptionellen Vorteile und Entwicklungsmöglichkeiten der Unternehmensform „eingetragene Genossenschaft" und ihre Verortung in einer alternativen „Solidarischen Ökonomie“(Voß2010; Altvater/Sekler 2006) ist in ihrer wissenschaftlichen Auseinandersetzung jedoch kein Diskurs des 21. Jahrhunderts.

Schon in den 1980er Jahren reklamierte Klaus Novy (1985) eine Renaissance der Genossenschaften. In der Tat hatte diese Entwicklung vor 30 Jahren ihren letzten Höhepunkt, als im Zusammenhang mit der Alternativbewegung der genossenschaftliche Gedanke erneut publiziert und die Genossenschaft als Unternehmensform wiederentdeckt wurde, sodass gar von einer neuen Genossenschaftsbewegung die Rede war. Gemeint waren damit die Initiativen der Selbstverwaltungswirtschaft, der sozialen Selbsthilfe, der Belegschaftsbetriebe und insbesondere die genossenschaftlich organisierte Wohnungswirtschaft (Bierbaum/Riege 1985). In diese Zeit fiel auch die Gründung von neuen genossenschaftlichen Organisationen wie z. B. des WohnBundes und des Bundesvereins zur Förderung des Genossenschaftsgedankens e.V., deren Interesse einer Revitalisierung der Genossenschaftsidee galt (innova 1990; Bierbaum/Riege 1989). Dies galt sogar in Zeiten der Wende der „Vergenossenschaftlichung Osteuropas“ (Novy 1990). Novy und seine Mitstreiter blieben aber in den 1980er und 90er Jahren eher einsame Rufer nach einer Wiederbelebung von Genossenschaftskultur und Wirtschaftsdemokratie. Lange Zeit war es recht still um die Genossenschaftsbewegung, was auch mit der Diskreditierung genossenschaftlichen Denkens durch politische Entscheidungsträger zusammenhing, die sich im Zuge der Ausweitung der Arbeiterbewegung gegen die Idee „einer positiven Ökonomie der Alternative“ stemmten, um den Hegemonialanspruch der Politik zu untermauern (Novy 1982, S. 121). Nicht zuletzt aufgrund des UN-Jahres der Genossenschaften und einer wieder auflebenden Kritik am gegenwärtigen kapitalistischen Wirtschaftssystem, die u. a. in der Occupy-Bewegung ihren Ausdruck findet (Graeber 2012), scheint die Diskussion um genossen-

Hier zitiert nach http://social.un.org/index/Cooperatives/ News/tabid/1496/news/157/Default.aspx. 
schaftliche Lösungen jedoch nun aus ihrem Dornröschenschlaf zu erwachen. ${ }^{2}$

Doch kann heute von einer Renaissance der Genossenschaften gesprochen werden? Und erleben wir damit auch eine Renaissance für „gemeinschaftlich dezentrale Reformansätze“ (Novy 1979), wie sie unter den Begriffen Wirtschaftsdemokratie (Martens 2010), alternatives Wirtschaften oder Alternativökonomie beschrieben werden? Aktuell treten in der deutschen Diskussion um Alternativen oder Ergänzungen zum kapitalistischen Wirtschafts- und Gesellschaftssystem noch weitere Begrifflichkeiten hinzu: die Solidarische Ökonomie (Altvater/Sekler 2006; Elsen 2011), die Gemeinwesenökonomie (Elsen 1998; Brinkmann 2010) und die Gemeinwohlökonomie (Felber 2010).

An diese kapitalismuskritischen bzw. -ergänzenden Stoßrichtungen schließt sich die dritte Frage an, die darauf gerichtet ist, ob Genossenschaften überhaupt als Teil dieser wirtschaftlichen Reformansätze verstanden werden können oder ob sie vielmehr fester Bestandteil des kapitalistischen Systems mit besserer Absicht sind, also gar als bessere oder als „soziale Kapitalisten“ (Koch 2007) gelten können.

Die erste Frage scheint sich anhand der quantitativen Fakten tatsächlich eindeutig mit „)a “beantworten zu lassen. Der Genossenschaftssektor ist mit rund 7.600 Unternehmen, 860.000 Beschäftigten und 20 Mio. Mitgliedern ein stabiler Wirtschaftsfaktor, was sich durch das anhaltend positive Neugründungsgeschehen seit ca. 2006 bestätigt. Jeder vierte Bundesbürger ist inzwischen Mitglied einer oder mehrerer Genossenschaften.

Die zweite Frage weist eher auf einen Defizitbereich der politischen und wirtschaftswissenschaftlichen Diskussion hin, den Klaus Novy (1979) schon früh kritisierte und der bis heute nicht an Gültigkeit verloren hat.

Um die zweite und dritte Frage fundiert und abschließend beantworten zu können, fehlt es noch an der entsprechenden Empirie. Trotzdem möchten wir an dieser Stelle einige Forschungsfragen aufwerfen und Arbeitshypothesen entwickeln, um damit einen Beitrag zur systematischen Analyse des Forschungsgegenstandes zu leisten.

Der Aufsatz beginnt damit, das Dickicht der Begrifflichkeiten zu entwirren und die Begriffsvielfalt alternativer Wirtschaftsformen miteinander in Beziehung zu setzen, die eine Verortung der Genossenschaften zwischen privatkapitalistischer Wirtschaft (Erster Sektor) und zivilgesellschaftlicher Wirtschaft (Dritter Sektor) möglich macht. Anschließend wird überblicksartig das empirische Feld der verschiedenen genossenschaftlichen Sparten in Deutschland aufgezeigt, um daraus Rückschlüsse auf den Stellenwert der Genossenschaften im Wirtschaftssystem der Bundesrepublik zu ziehen und erste Befunde und Entwicklungstrends abzuleiten. Nach einem Einblick in das Gründungsgeschehen insgesamt werden die Gründe für den Boom von Energiegenossenschaften nachgezeichnet, erste Trends benannt und Fragen hinsichtlich der Verortung in den vorgestellten Alternativformen des Wirtschaftens aufgeworfen.

\section{Begriffe zur alternativen Wirtschaft}

\subsection{Genossenschaften und sozialreformerische Genossenschaften}

"Genossenschaften sind Gesellschaften von nicht geschlossener Mitgliederzahl, deren Zweck darauf ausgerichtet ist, den Erwerb oder die Wirtschaft ihrer Mitglieder oder deren soziale und kulturelle Belange durch gemeinschaftlichen Geschäftsbetrieb zu fördern“' (Geschwandtner/Helios 2006, S.37); so wird die eingetragene Genossenschaft in $\$ 1$ des Genossenschaftsgesetzes definiert. Hinsichtlich der Genossenschaftlichkeit von Unternehmen gilt es, zwischen der rechtlichen Frage und der sozialen Organisation zu unterscheiden. Denn nicht jede eingetragene Genossenschaft ist auch von ihrer sozialen Ausgestaltung als genossenschaftlich zu charakterisieren. Umgekehrt gibt es zahlreiche Unternehmen, die als Genossenschaften bezeichnet werden können, auch wenn diese nicht die eG als Rechtsform gewählt haben. Insofern erscheint es sinnvoll, deren Genossenschaftlichkeit anhand der vier wesentlichen Charakteristika einer Genossenschaft zu reflektieren: dem Förder-, dem Identitäts-, dem Demokratie- und dem Solidaritätsprinzip (Klemisch/Flieger 2007, S. 5).

(1) Förderwirtschaftliches Agieren ist ein entscheidendes Charakteristikum genossenschaftlicher Unternehmen. Der förderwirtschaftliche Auftrag wurde für eingetragene Genossenschaften sogar im Gesetz verankert. Er besagt: Nicht die Verwertung von Kapital und das Erwirtschaften von Gewinn soll Hauptzweck einer Genossenschaft sein, sondern die Förderung der Mitglieder in dem Geschäftsfeld, in dem sie angesiedelt ist.

(2) Das empirisch am leichtesten überprüfbare genossenschaftliche Prinzip und somit das eindeutigste Erkennungsmerkmal stellt das Identitätsprinzip dar (Laurinkari/Brazda 1990, S. 70ff.; Eschenburg 1971, S. 6). Zwei Rollen, die sich sonst am Markt gegenüberstehen, fallen in der Personengruppe der Genossenschaftler zusammen. Bei der Wohnungsbaugenossenschaft sind es Mieter und Vermieter, in der Konsumgenossenschaft Verbraucher und Händler, in der Produktivgenossenschaft Kapitaleigner und Beschäftigte.

(3) Mit dem dritten Prinzip, dem Demokratieprinzip „ein Mensch eine Stimme“, werden Genossenschaften am

\footnotetext{
2 So ist in der Bundestagsfraktion der SPD ein Antrag zum Thema „Genossenschaftsgründungen erleichtern und fördern" im Umlauf. Die Parlamentarische Linke in der SPD veranstaltete bereits mehrere Diskussionsrunden und veröffentlichte einen Reader zum Thema: „Genossenschaften eine andere Form des Wirtschaftens" (Vogt 2011), und die Friedrich-Ebert-Stiftung führt in diesem Jahr eine Veranstaltungsreihe zum Thema durch.
} 
stärksten verbunden (Winter/Mändle 1980, S. 256f.). Unabhängig von der Anzahl der eingebrachten Kapitalanteile, der Erfahrungen oder der Position im Betrieb verfügt jedes Mitglied in der Generalversammlung formal über das gleiche Stimmrecht.

(4) Das Solidaritätsprinzip als viertes Prinzip soll an dieser Stelle besonders betont werden, auch wenn es unter Genossenschaftstheoretikern als das umstrittenste gilt. Bekannter ist der Begriff "Genossenschaftsgeist" (Hettlage 1990, S. 123ff.). Mit diesem Prinzip wird das Thema Unternehmenskultur in besonderem Maße tangiert, geht es hierbei doch um die Ausprägung genossenschaftsspezifischer Werte, Einstellungen und Verhaltensweisen. Durch die Betonung und konsequente Anwendung bestimmter Werte und der damit verbundenen Verhaltensweisen kann in genossenschaftlichen Unternehmen eine höhere Stabilität erreicht werden. Das gilt vor allem für die Gründungsphase der Genossenschaft, aber auch für die Zeiten intensiver sozialer Konflikte oder wirtschaftlicher Turbulenzen. Kommt hier das Solidaritätsprinzip nicht zum Tragen, besteht die Gefahr, dass eine Gründung scheitert oder die betreffende Genossenschaft in Krisenzeiten relativ schnell auseinanderfällt.

In Deutschland hat es historisch schon immer zwei Strömungen innerhalb der Genossenschaftsbewegung gegeben, die in der von 1902 bis 1933 gespaltenen Geschichte der Genossenschaftsverbände ihren realen Ausdruck fanden und die entscheidenden Einfluss auf die Frage der Verortbarkeit von Genossenschaften innerhalb der Diskussion um dezentrale Reformansätze hat: Für Mersmann und Novy (1991, S. 29f.) gelten Genossenschaften erst dann als sozialreformerisch, „, [...] wenn sie nicht bloß der Besserstellung einer Sondergruppe dienen, wenn umgekehrt also die wirtschaftlichen Vorteile der genossenschaftlichen Organisation prinzipiell verallgemeinerungsfähig sind. [...] [S]ozialreformerische Genossenschaften verstehen sich als Substitute zu Privateigentum und Marktwirtschaft (,Kooperation statt Konkurrenz', ,Solidarismus')“.

\subsection{Genossenschaften - Teil der solidarischen Ökonomie oder Gemeinwesenökonomie?}

In der wissenschaftlichen Diskussion um den Begriff solidarische Ökonomie und deren Umsetzung in die wirtschaftliche Praxis spielen sozialreformerische Genossenschaften eine bedeutsame Rolle (Kerber-Klasen 2012, S. 283). Die „Solidarische Ökonomie“ wird als Sammelbegriff für die vielfältigen Formen der alternativen Ökonomie gebraucht. In Deutschland hat dieses Konzept durch den von Attac im Herbst 2006 unter dem gleichnamigen Titel durchgeführten Kongress neue Prägekraft erfahren (Altvater/Sekler 2006). Seitdem subsumiert die solidarische Ökonomie, die aus der lateinamerikanischen alternativ-ökonomischen Diskussion stammt, unter sich sowohl sozialreformerische Produktivgenossenschaften als auch andere Formen alternativen Wirtschaftens. Gemeint sind damit u. a. Energiegenossenschaften,
Dorfläden, Konsumgenossenschaften, fairer Handel, Wohnprojekte, Gemeinschaftsgärten, aber auch Tauschringe oder Regionalwährungen (Übersichten bieten Giegold/Embshoff 2008; Verein zur Förderung der solidarischen Ökonomie 2011; Voß 2010; Notz 2011; vgl. auch Kerber-Clasen 2012, S. 283). Entscheidend hierbei sind die Affinitäten zwischen einer genossenschaftlich orientierten Arbeitsweise und den verschiedenen Formen der alternativ-ökonomischen Produktionsstrategien. So weisen die Organisations- und Handlungsprinzipien Freiwilligkeit, Solidarität, Kooperation, Demokratie, Selbstorganisation und Gemeinwohlorientierung eine hohe Identität mit den genossenschaftlichen Organisationsprinzipien auf (Elsen 2011, S. 93ff.).

Giegold und Embshoff(2008, S. 12ff.), zwei Protagonisten dieses Diskussionszusammenhangs, verstehen darunter Formen des Wirtschaftens, die menschliche Bedürfnisse auf der Basis freiwilliger Kooperationen, Selbstorganisation und gegenseitiger Hilfe befriedigen. Auch Altvater (2012, S. 55) stellt fest, dass ein „nicht eng definierter Begriff der solidarischen Ökonomie möglicherweise der angemessenere [ist], um die Vielfalt des genossenschaftlichen und gemeinschaftlichen Wirtschaftens erfassen zu können“.

Gemeinsam ist den diskutierten Begriffsbestimmungen solidarischer Ökonomie (z. B. Elsen 2011; Giegold/Embshoff 2008; Müller-Plantenberg 2007), „dass diese ein ideelles und/oder materielles Gegenmodell zu einem neoliberalen kapitalistischen Wirtschafts- und Gesellschaftssystem entwerfen, in dem der Profitlogik idealtypisch andere Prinzipien des Wirtschaftens gegenübergestellt werden“ (KerberClasen 2012, S. 283).

Große Übereinstimmung weist die Begrifflichkeit der Solidarischen Ökonomie mit den in Deutschland unter dem Begriff Gemeinwesenökonomie vertretenen Ansätzen auf. Bezogen auf ihre sozialen und politischen Ziele und auf ihre Arbeitsorganisation lassen sich Gemeinwesenökonomien der „Économie Sociale“ und somit dem „dritten Sektor“ zwischen Staat und Markt zuordnen (Elsen 1998, S.81). Da Genossenschaften Selbsthilfeorganisationen sind, die auf den Prinzipien der Selbstverwaltung und Selbstverantwortung beruhen, können sie somit als natürlicher Teil der solidarischen oder Gemeinwohlökonomie beschrieben werden (Brinkmann 2010, S. 234 und Elsen 1998). Ob dies für alle Genossenschaften gilt, bleibt allerdings offen.

\subsection{Modell der Gemeinwohlökonomie}

Ein weiteres aktuell diskutiertes Konzept, das ebenfalls Implikationen zur Solidarischen Ökonomie aufweist, ist das von Christian Felber (2010) skizzierte Modell der Gemeinwohlökonomie. Das Konzept stammt aus dem Diskussionszusammenhang von Attac Österreich, zu deren Mitgründern Felber zählt. Die Gemeinwohlökonomie wird hier den sogenannten Irrwegen von Realsozialismus und Kapitalismus als Dritter Weg gegenübergestellt. Ohne auf den Genossenschaftsbegriff zu rekurrieren, werden genossenschaftliche Prinzipien wie Solidarität, Soziale Gerechtigkeit 
und demokratische Mitbestimmung als Handlungsmaximen herausgearbeitet. Unternehmen können sich einer Gemeinwohlbilanz unterziehen, die Parallelen zu einer Sozialbilanz oder den Prinzipien der Corporate Social Responsibility (CSR) aufweist (vgl. u. a. Müller/Schaltegger 2008). Felbers Kritik an einer rein auf extrinsischen Motivlagen beruhenden Konkurrenzwirtschaft basiert auf der hohen Diskrepanz zwischen Werten des alltäglichen Lebens wie Solidarität, Nächstenliebe und Altruismus und den in kapitalistischer Produktionsweise eingeforderten Eigenschaften wie Egoismus, Geiz und Gier (Felber 2010, S.16). ${ }^{3}$

Felber bezieht die Gemeinwohlökonomie u. a. auf die verfassungsmäßige Verankerung einer moralischen Verantwortung des Wirtschaftens, wie sie in einigen Länderverfassungen festgelegt wurde: „Alle wirtschaftliche Tätigkeit dient dem Gemeinwohl“ (Bayerische Verfassung Art. 151). Hiermit wäre dann auch ein Pfad gelegt in den Bereich der Wirtschaftsdemokratie, der in der deutschen Nachkriegsdiskussion seit Viktor Agartz (Bispinck et al. 2008) keine Rolle mehr spielte, weil Fragen der Wirtschaftsdemokratie auf die Umsetzung von betrieblicher Mitbestimmung und branchenbezogener Tarifpolitik verkürzt wurden, ohne wirtschaftspolitische Zielsetzungen, die über die Unternehmensebene hinausgehen, oder Elemente einer regionalen Wirtschaftsdemokratie in den Blick zu nehmen (Martens 2010; Demirovic 2007, 2011). Die Finanz- und Bankenkrise hat zumindest auf der Ebene der theoretischen Auseinandersetzung diese Diskussion in kritischer Absicht wieder befördert. Sedlacek (2012) und Nida-Rümelin (2012) diskutieren ökonomische Fragen vor dem Hintergrund von Ethik und Moral, allerdings ohne sich in die Niederungen der wirtschaftlichen oder wirtschaftspolitischen Praxis zu begeben und ohne die Konzepte zur Unternehmensform der Genossenschaft in Relation zu setzen.

Um der Frage nach einer Renaissance der Genossenschaft nachzugehen und ihren sozialreformerischen Charakter zu beleuchten, werden zunächst der Status quo sowie die verschiedenen Typen und Geschäftsfelder von Genossenschaften betrachtet.

\section{Status quo und Typen - Überblick über die Sparten und aktuelle Entwicklung in Genossenschaften}

Im Zusammenhang der bundesrepublikanischen Wirtschaft mit fast 3,6 Mio. Unternehmen und 25,2 Mio. sozialversicherungspflichtig Beschäftigten ist der Genossenschaftssektor (Tabelle 1) mit ca. 7.600 Unternehmen, ca. 860.000 Beschäftigten und 20,7 Mio. Mitgliedern ein zwar kleiner aber stabiler Wirtschaftsfaktor (Stappel 2011).

In der Verbandsstatistik werden fünf Genossenschaftssektoren beschrieben (Übersicht 1). Dabei handelt es sich um Genossenschaftsbanken, ländliche, gewerbliche, Konsum- und Wohnungsgenossenschaften. Diese sind in ihren Geschäftsfeldern und ihrer Charakteristik sehr unterschiedlich und sollen daher näher beleuchtet werden.

\subsection{Konsumgenossenschaften: zwischen Gemeinwirtschaft und Nischendasein}

Die Konsum- bzw. Verbrauchergenossenschaften sind eine besondere Form der Genossenschaften im Einzelhandel, die sich in erster Linie mit dem Vertrieb von Nahrungs- und Genussmitteln sowie verwandten Waren des täglichen Bedarfs befassen. Sie entstanden ursprünglich auf Initiative von Verbrauchern, die sich von den etablierten Anbietern ausgebeutet fühlten und ihre Lebenshaltung durch billigere, beziehungsweise effizientere Warenversorgung zu verbessern suchten. Heute gibt es noch 33 Konsumgenossenschaften im Lebensmittelbereich. Etwa zehn wirtschaften noch unter dem Label coop. Zu ihnen gehört auch die coop Schleswig-Holstein als letzte große Verbrauchergenossenschaft in Deutschland. Eine kleine, bescheidene Renaissance erfuhr die Idee in den 1980er Jahren mit der Gründung zahlreicher Erzeuger-Verbraucher-Gemeinschaften, von denen sich heute noch knapp zehn in der Rechtsform der Genossenschaft für einen verantwortungsvollen Umgang mit der Natur und eine solidarischen Zusammenarbeit mit den Erzeugern engagieren (Asendorf et al., S. 2003). Im Unterschied zu den rund 30 Dorfladengenossenschaften in Deutschland vertreten sie explizit politische Zielsetzungen, dagegen setzen Letztere vor allem auf den Zusammenhalt der dörflichen Gemeinschaft.

\subsection{Wohnungsbaugenossenschaften - Solides Fundament für neue Herausforderungen}

„Aufgabe der Wohnungsbaugenossenschaften ist die wohnliche Versorgung ihrer Mitglieder durch Überlassung von Wohnungen in allen Rechts- und Nutzungsformen, die Hilfe bei der Bildung von Eigentum an Haus und Wohnung, die Verbesserung ihrer Wohnverhältnisse durch wohnungswirtschaftliche Leistungen und die Wohnversorgung ergänzende Maßnahmen“ (Jenkis 1992, S. 734; Mändle 2005, S. 256f.). Darunter sind alle Nutzungsformen vom Mietwohnungsbau bis zur Schaffung von gemeinschaftlichem oder individuellem Wohneigentum zu verstehen. Bezeichnungen wie Mietergenossenschaften, Baugenossenschaften, Siedlungsgenossenschaften, Bestandsgenossenschaften etc. verdeutlichen, wie breit das Spektrum der Wohnungsbaugenossenschaften ist, ohne dass hier eine ausreichend differenzierte Typologie ihrer Umsetzungsformen vorliegt.

(3) Interessant ist, dass sich hier offenbar eine Bewegung auf den Weg gemacht hat, die von etlichen Unternehmen und nicht nur in Genossenschaften umgesetzt wird. Bisher unterstützen 592 Firmen das Konzept (taz vom 14/15.4., S.21). 
Genossenschaftliche Unternehmen 2000 bis 2010

\begin{tabular}{|c|c|c|c|c|c|}
\hline Jahresende & 2000 & 2002 & 2004 & 2005 & 2010 \\
\hline Zahl der Unternehmen & 9.094 & 8.332 & 7.936 & 7.763 & 7.619 \\
\hline Mitglieder & 20.074 .000 & 20.117 .000 & 19.965 .000 & 20.110 .000 & 20.744 .000 \\
\hline Mitarbeiter & 463.000 & 443.800 & $732.500 * *$ & 758.300 & 862.500 \\
\hline
\end{tabular}

** seit 2004 einschließlich Edeka und REWE-Gruppe, der Wachstumssprung bei den Beschäftigtenzahlen zwischen 2002 und 2004 ist allein auf die zusätzliche Erfassung zurückzuführen.

Quelle: Zusammenstellung der Autoren nach Stappel (2006; 2011).

\section{Genossenschaften nach Verbandsstatistik Ende 2010}

\begin{tabular}{l|l}
\hline Konsumgenossenschaften & 33 mit 355.000 Genossen und 14.330 Beschäftigten \\
Wohnungsbaugenossenschaften & 1.931 mit 2,82 Mio. Genossen und 23.600 Beschäftigten \\
Gewerbliche Genossenschaften & 2.018 mit 315.000 Genossen und 543.000 Beschäftigten \\
Genossenschaftsbanken & 1.157 mit 16,68 Mio. Genossen und 186.900 Beschäftigten \\
Ländliche Genossenschaften & 2.480 mit 563.000 Genossen und 84.120 Beschäftigten \\
\hline
\end{tabular}

Quelle: Stappel (2011, S. 10).

Nach der Studie von Theurl/Wendler (2011, S. 85) wissen 72,3 \% der Deutschen, dass in der Wohnungswirtschaft Genossenschaften tätig sind. Damit sind Wohnungsgenossenschaften eine bekannte Genossenschaftssparte. Sie bewirtschaften 2,3 Mio. Wohnungen und verfügen damit über $10 \%$ des Mietwohnungsbestandes (Stappel 2011, S. 36). Kleine Genossenschaften mit bis zu 500 Mietwohnungen stellen dabei den größten Anteil (GdW 2012).

Die Vergleichsbilanz von Genossenschaften und dem übrigen Wohnungsbestand Wohnungsmarkt kann sich sehen lassen: 5,70 zu 6,37€ Mietvergleich pro qm, 1,05 zu $1,25 €$ als warme Betriebskosten (61\% der Gebäude sind energetisch saniert), 4,4 zu 8,6\% Leerstandquote, 9,1 zu 10,1 \% Fluktuationsquote (ebd.).

Die kleinen Genossenschaften sind oft vom ehrenamtlichen Engagement des Vorstands geprägt. Für Wohnungsgenossenschaften spricht ihre Tradition, es gibt lange Bewerberlisten und die Insolvenzquote liegt bei Null. Sie scheinen gut aufgestellt, um die größten Herausforderungen im Wohnungssektor - Energiewende und demografischen Wandel - zu meistern.

\subsection{Gewerbliche Genossenschaften und Produktivgenossenschaften}

Gewerbliche Genossenschaften sind in den unterschiedlichsten Branchen tätig. Schwerpunkte liegen im Handel, Handwerk und im Verkehrssektor. In der offiziellen Genossenschaftsstatistik werden Produktivgenossenschaften den gewerblichen Genossenschaften zugerechnet (Stappel 2011, S. 26). Sie unterscheiden sich in ihrer Struktur und Zielsetzung wesentlich von den übrigen Genossenschaftsarten.

Es handelt sich hierbei um Vollgenossenschaften zur Verwertung der Arbeitskraft ihrer Mitglieder. Alle anderen sind sogenannte Hilfs- oder Ergänzungsgenossenschaften im Dienst der unabhängigen Einzelwirtschaften ihrer Mitglieder. Als Grundtypen lassen sich die klassischen Produktivgenossenschaften, die konsequenten Beteiligungsunternehmen und die Selbstverwaltungsbetriebe identifizieren (Flieger 2006, S. 54; Atmaca 2007, S. 549). Sie sind jeweils vor dem Hintergrund unterschiedlicher Werte und historischer Prägungen entstanden. Angaben zur Gesamtzahl produktivgenossenschaftlich strukturierter Betriebe variieren stark. Sie kann in Deutschland auf etwa 5.000 geschätzt werden (Flieger 2006). Dabei handelt es sich nicht unbedingt um eingetragene Genossenschaften, sondern um produktivgenossenschaftlich strukturierte Unternehmen. Damit sind Selbstverwaltungsbetriebe und Mitarbeiterbeteiligungsunternehmen mit über 50 \% Beteiligung der Beschäftigten gemeint.

Von den abweichend zur Verbandserhebung geschätzten etwa 800 Produktivgenossenschaften, die die genossenschaftliche Rechtsform nutzen, sind die meisten der klassischen Produktivgenossenschaft zuzuordnen (Flieger 2006). Die Tätigkeitsfelder dieser Produktivgenossenschaften liegen vor allem in handwerklichen, manufakturellen bzw. semiindustriellen Branchen und Sektoren (Atmaca 2007, S. 548ff.). 


\subsection{Genossenschaftsbanken - marktmächtig und wertebasiert}

Den Bankgenossenschaften kommt eine herausragende Bedeutung im deutschen Genossenschaftswesen zu. 1.121 Genossenschaftsbanken sind mit rund 160.000 Beschäftigten, 13.350 Bankstellen und 17 Mio. Mitgliedern der marktmächtigste Sektor der traditionellen Genossenschaften. Sie stellen damit doppelt so viele Mitglieder wie alle anderen Genossenschaften in Deutschland zusammen. Der genossenschaftliche Finanzverbund besteht aus zwei Zentralbanken, sechs sogenannten Allfinanzpartnern wie der Bausparkasse Schwäbisch Hall, sechs Produktionspartnern wie dem DG Verlag und der Basis von 1156 Kreditgenossenschaften. Die durchschnittliche Mitgliederzahl dieser Genossenschaftsbanken liegt bei 14.000 und damit deutlich über der der sonstigen Genossenschaften. Diese genossenschaftliche Sparte verzeichnet den stärksten Mitgliederzuwachs. Allein im Jahr 2010 kamen knapp 300.000 neue Bankanteilseigner hinzu.

Genossenschaftsbanken sind ,ein Modell mit vielen Facetten“ (taz vom 28./29.01.2012). Genossenschaften gelten in der aktuellen Finanzmarktkrise als eine Unternehmensform von bemerkenswerter Stabilität. So gehören die beiden genossenschaftlichen Zentralbanken (DZ BANK und der WGZ BANK) zu den 25 größten Banken in Deutschland, die ohne staatliche Beteiligung arbeiten und die Volks- und Raiffeisenbanken haben in der aktuellen Krise ihr ohnehin gut dotiertes Eigenkapital noch erhöhen können. Die genossenschaftliche Gruppe als Verbundstruktur kann durch ihre Ausrichtung an Subsidiarität, demokratischer Willensbildung und ihren Sicherungseinrichtungen als ein Modell für funktionierende Selbstregulierung mit einem engen Bezug zur Realwirtschaft und Regionalität charakterisiert werden. Genossenschaftliches Banking ist idealtypischerweise wertebasiert und verknüpft Verbraucherschutz, Mitgliederorientierung und Kundennutzen. Genossenschaftsbanken haben allerdings ein Imageproblem: Sie sind zwar bei $64 \%$ der deutschen Bevölkerung bekannt (Theurl/ Wendler 2011, S. 85), gelten aber als altbacken, lieb und bodenständig.

\subsection{Ländliche Genossenschaften - Mehrwert für die Region}

Unter ländliche Genossenschaften subsumieren wir die Agrargenossenschaften, aber auch Raiffeisengenossenschaften, die als zentrale Handelspartner der Landwirtschaft auftreten. Agrargenossenschaften verzeichnen heute rund 37.000 Mitglieder und 22.000 Beschäftigte.

Die Wirtschaftskraft der Genossenschaften im Landwirtschaftssektor wird allgemein unterschätzt. Im Landwirtschaftssektor existierten 2010 über 2.000 Genossenschaften, nahezu gleich verteilt in den alten und neuen Bundesländern (1082 in den alten und 985 in den neuen Bundesländern) (Stappel 2011, S. 22ff.). Die Agrargenossenschaften als Nachfolger der ehemaligen landwirtschaftlichen Produktionsge- nossenschaften haben ca. 41.000 Mitglieder, 23.000 Mitarbeitende und verzeichnen einen Umsatz von ca. 1,8 Mrd. $€$. Damit erwirtschaften sie ca. 27 \% des Umsatzes der Landwirtschaft in den neuen Bundesländern. Gerade ländliche Genossenschaften schaffen hier einen Mehrwert für die Region und tragen zur Lösung sozialer, kommunaler und arbeitsmarkpolitischer Probleme bei.

Vor diesem Hintergrund können die Genossenschaften insgesamt als kleiner, aber stabiler Teil des ersten Sektors charakterisiert werden. Ob es sich dabei zumindest partiell um gemeinschaftlich dezentrale Reformansätze handelt und somit um Übergänge zum Dritten Sektor im Sinne einer solidarischen oder Gemeinwesenökonomie, lässt sich empirisch bisher kaum erfassen. Brinkmann (2010, S. 236) folgend kämen dabei Selbsthilfe-, Professions-, Erzeuger/ Verbraucher-, Wohnungsgenossenschaften sowie genossenschaftliche Auffanglösungen in die engere Wahl. Die jeweilige Ausrichtung der Motivlage von Genossenschaften am zivilgesellschaftlichen Engagement (Alscher 2011) könnte weitere Anhaltpunkte für eine Ausdifferenzierung von Genossenschaften zwischen erstem und drittem Sektor bieten.

\section{Aktuelle Befunde und Entwicklungs- trends - Neue Geschäftsfelder für Genossenschaften}

Zu den markantesten Trends in der Genossenschaftsentwicklung gehören ein lang anhaltender Konzentrationsprozess, eine zunehmende Internationalisierung und die Wiederentdeckung der Genossenschaft als Rechtsform für Neugründungen. Der Konzentrationsprozess vollzieht sich vor allem bei den Genossenschaftsbanken, aber auch bei den ländlichen und bestimmten gewerblichen Genossenschaften und ist Resultat von Fusionen in diesen Sektoren. Von 1960 bis heute ist die Zahl der Genossenschaften von ca. 27.000 in den alten Bundesländern auf heute ca. 7.600 in Gesamtdeutschland zurückgegangen. Dieser Prozess ist erst in den letzten Jahren gestoppt worden. So konnten im vergangenen Jahrzehnt immerhin ca. 1.200 Neugründungen registriert werden (Stappel 2011, S. 48).

Über die Gründe für diesen neuen Trend, der teilweise schon als Boom bezeichnet wird, lässt sich trefflich streiten. Eine fundierte Analyse, die über die Darstellung von Fallbeispielen hinausgeht, fehlt bislang (Münkner/Ringle 2010; Göler von Ravensburg 2011). Die Genossenschaftsverbände schreiben die positive Entwicklung gerne ihren eigenen Neugründungsinitiativen zu (Stappel 2011, S. 4). Die Politik ist eher geneigt, die Novellierung des Genossenschaftsgesetzes von 2006 und die damit verbundenen Erleichterungen der Gründung als Hauptgrund anzuführen. ${ }^{\circ}$

Einen eindeutigen empirischen Beleg gibt es für beide Positionen derzeit nicht (Grosskopf et al. 2012, S. 26ff.). 
TABELLE 2

\section{Neugründungen von Genossenschaften 2001 bis 2010}

\begin{tabular}{|c|c|}
\hline Art der Genossenschaften & Anzahl der Neugründungen \\
\hline Gewerbliche Genossenschaften & 1.064 \\
\hline - davon Energiegenossenschaften & (273) \\
\hline Ländliche Genossenschaften & 85 \\
\hline - davon Bioenergiegenossenschaften & (20) \\
\hline Wohnungsgenossenschaften & 89 \\
\hline Konsumgenossenschaften & 1 \\
\hline Genossenschaftsbanken & 0 \\
\hline Insgesamt & 1.239 \\
\hline
\end{tabular}

Anzunehmen ist vielmehr, dass die Erschließung von neuen Geschäftsfeldern und das Kommunizieren von guter Praxis und wirtschaftlichen Erfolgen zu Nachahmungseffekten bei Genossenschaftsgründungen führen (Stappel 2011, S. 79). Diese Entwicklung wird unterstützt, wenn Genossenschaften zusätzlich auf bestimmte Förderstrukturen, wie z. B. im Umweltbereich oder im Bereich der erneuerbaren Energien, zurückgreifen können. Grundsätzlich ist die Bedeutung des Gegenstandsbereichs Neugründungen in der Genossenschaftsforschung und -praxis erkannt, denn insbesondere das Gründungsverhalten gibt Hinweise auf geeignete innovative Geschäftsfelder und die Akzeptanz der Unternehmensform (Münkner/Ringle 2010; Göler von Ravensburg 2011).

Die meisten Neugründungen (Tabelle 2) im letzten Jahrzehnt kamen im Bereich der gewerblichen Genossenschaften zustande. Der Schwerpunkt lag bei der Energie (25\%) und den übrigen Dienstleistungen (20\%), die sich sehr vielfältig von Architektur- über Ingenieurbüros bis hin zu Werbe- und Multimedia Agenturen verteilen. Erklärt wird dieses Phänomen mit der Fähigkeit von Genossenschaften, Marktunzulänglichkeiten zu kompensieren. Dies trifft auch auf Gründungen im Gesundheitssektor (12\%), im sozialen Sektor (7 \%), beim Erhalt kommunaler Aufgaben und Infrastruktur (3\%), für zwischenbetriebliche Kooperationen und Unternehmensnachfolgen im Handwerk (3\%) oder Belegschaftsinitiativen zur Erhaltung von Betrieben zu (Klemisch/Maron 2010).

Grundsätzlich soll aber die konstatierte Neugründungsdynamik nicht über eine schlechte Performance der Genossenschaften (Blome-Drees 2010) und ihren relativ geringen Bekanntheitsgrad hinwegtäuschen. Verglichen mit der Zahl der Unternehmensgründung von jährlich insgesamt 400.000 bis 500.000, ist die Zahl der neu gegründeten Genossenschaften eine Marginalie. Genossenschaften liegen weder im Mainstream der gesellschaftlichen Diskussion, noch sind sie in der Alltagswelt präsent. So kann es auch nicht überraschen, dass Theurl/Wendler (2011, S.19) in ihrer Studie insbesondere bei der jungen Generation einen deutlichen Abfall des Bekanntheitsgrades von Genossenschaften feststellten. ${ }^{\ominus}$ Die zu beobachtende Gründungswelle im Geschäftsfeld der Energiewirtschaft macht jedoch Hoffnung, dass genossenschaftlich organisiertes Wirtschaften wieder an Reputation gewinnt.

\section{Gründungswelle in neuen Geschäfts- feldern - Das Beispiel der Energie- genossenschaften}

Kann die quantitative Entwicklung genossenschaftlich organisierter Unternehmen angesichts von über 600 neu gegründeter Genossenschaften in den Jahren 2010 und 2011 mit Blick auf eine „Renaissance der Genossenschaften“ zumindest vorsichtig optimistisch stimmen, so gilt dies für das Geschäftsfeld der Energiewirtschaft verstärkt.

\subsection{Energiegenossenschaften als Teil der Energiewende}

Das Neugründungsgeschehen ist im Bereich der erneuerbaren Energien besonders dynamisch. Engagierte Bürger schließen sich zu Energiegenossenschaften zusammen, um gemeinsam Solar-, Biogas- oder Windkraftanlagen zu betreiben. Mittlerweile belegt eine Fülle von aktuellen Beispielen, dass die regionale Daseinsvorsorge von den Bürgern vor Ort durchaus in Eigenregie zu übernehmen und erfolgreich zu gestalten ist (Klemisch/Maron 2010). In einer aktuell durchgeführten Vollerhebung ermittelte das Klaus Novy Institut zum Stichtag 31.12.2011 586 eingetragene Genossenschaften, die ausschließlich energiewirtschaftlich tätig sind. Auffällig ist, dass die Mehrzahl dieser Genossenschaften innerhalb der letzten fünf Jahre gegründet wurde; allein 2011 wurden in den Registergerichten mindestens 196 Gründungen verzeichnet.

Energiegenossenschaften sind kein neues Phänomen. Lokal operierende Elektrizitätsgenossenschaften übernahmen vorzugsweise im ländlichen Raum schon im 19. Jahrhundert die Aufgabe der Stromerzeugung und Stromverteilung (Flieger/Klemisch 2008, S. 107f.). Historisch sind Genossenschaften geradezu klassische Infrastrukturleis-

4 Durch die Novellierung wurde neben einer Erleichterung von Gründung und Prüfung auch ausdrücklich eine Erweiterung der Zwecksetzung auf soziale und kulturelle Belange verankert.

(5) Die Autorinnen bieten hierzu keine Erklärung an. Zu vermuten ist aber, dass Genossenschaften in der Lebenswelt inklusive Schule, Ausbildung und Studium der unter 30-Jährigen eine immer geringere Rolle spielen. 
tungsträger. So gab es 1910 in der Schweiz ca. 1.500 Energiewirtschaftsgenossenschaften. In den ländlichen Gebieten Deutschlands war es in erster Linie die fehlende wirtschaftliche Rentabilität für größere Stromanbieter, die die Bevölkerung zur genossenschaftlichen Selbsthilfe zwang: Die Energieversorgung der Region wurde in die eigene Hand genommen (ebd.; Volz 2011, S. 3).

Der anhaltende Boom von Energiegenossenschaften aus dem Bereich der erneuerbaren Energien ist hingegen weniger mit der Notwendigkeit der Aufrechterhaltung der eigenen Versorgungssicherheit zu erklären; die infrastrukturellen Voraussetzungen für die ländliche Energieversorgung durch die konventionellen Energieversorger sind mittlerweile in weiten Teilen vorhanden. Das genossenschaftliche Grundprinzip der Selbsthilfe bezieht sich bei neu gegründeten Energiegenossenschaften eher auf das Fehlen einer klimaverträglichen, aber gleichzeitig kostengünstigen Energieversorgung (Flieger 2011, S. 59).

Die Dynamisierung des genossenschaftlichen Neugründungsgeschehens muss daher im Kontext der in Deutschland eingeleiteten Energiewende gesehen werden. Energiegenossenschaften sind Teil des sozialökologischen Modells eines Umbaus der Energiewirtschaft, der den Ausbau der erneuerbaren Energien befördert (Mautz/Rosenbaum 2012, S. 87). Nach dem Willen der Bundesregierung soll der Anteil der erneuerbaren Energien am gesamten Stromverbrauch bis zum Jahr 2050 auf mindestens $80 \%$ gesteigert werden (BMU 2011, S. 9) - ein ehrgeiziges Ziel, das mit Blick auf die bisher genutzte und die potenziell nutzbare Anschlussleistung an Energie aus regenerativen Quellen jedoch nicht als reine Utopie zu bezeichnen ist.

Aus der Abhängigkeit zwischen geologischer Standortbeschaffenheit und der Raumbedeutsamkeit regenerativer Energieträger führt diese Umgestaltung der Strom- und Energieversorgung zwangsläufig zu einer Dezentralisierung der energetischen Produktion- und Versorgungsleistungen (Scheer 2010). Verbunden mit dem dezentralen Charakter lokal agierender Energiegenossenschaften ergibt sich somit die Chance, die bisherige Struktur, die von der Vormachtstellung der vier großen Energieversorger geprägt ist (vgl. dazu Bontrup/Marquard 2010, S.180f.), zu entflechten und durch dezentral angebundene Anlagen gleichzeitig eine regionale Wertschöpfung zu generieren. Untermauert wird die Hoffnung auf eine strukturelle Veränderung der Energieversorgung durch die Eigentümerstruktur im Bereich der erneuerbaren Energien. Denn diese wird geprägt durch das Eigentum von Privatpersonen, während die großen vier Energieversorgungsunternehmen nur eine untergeordnete Rolle spielen (trend:research 2011).

\subsection{Energiegenossenschaften - Ausdruck von Bürgerengagement und Partizipation}

Die Energiewende wird in erster Linie durch Investitionen, das Engagement und die Partizipationsbereitschaft der Bür- ger getragen. Die Umsetzung wird aber in den Genossenschaften selbst als risikobehafteter Weg eingeschätzt.

Die strukturelle Veränderung hin zu einer dezentraleren Energieversorgung ist der eine, die Notwendigkeit der gesellschaftlichen Akzeptanz einer breiten Bevölkerungsschicht beim Bau von Erneuerbare-Energie-Anlagen und Stromtrassen ein weiterer Aspekt, der für einen genossenschaftlich organisierten Umbau der Energiewirtschaft spricht (Schweizer-Ries 2010, S. 2). Dabei ist die gesellschaftliche Akzeptanz der Energiewende in erster Linie von den bevorzugten Umsetzungsprozessen der verantwortlichen Akteure abhängig. Hierbei zeigt sich, dass die Befürwortung regenerativer Energietechnologien, verstanden als „[...] das positive, zeitlich relativ konstante Ergebnis eines an bestimmte Rahmenbedingungen (Einflussfaktoren) geknüpften Bewertungsprozesses der jeweiligen Technologie durch ein Akzeptanzsubjekt [...]“ (ebd., S. 12) stark mit den vorhandenen Beteiligungsmöglichkeiten der Bürgerinnen und Bürger korreliert. „Die Bereitschaft sich einzubringen und aktiv zu beteiligen, ist also nicht nur bei einem Teil der Bevölkerung vorhanden, sondern ist vielerorts ein wesentlicher Baustein, teilweise sogar die Basis regionaler Erneuerbare-Energie-Prozesse.“ (ebd., S. 9)

Damit werden Energiegenossenschaften zu einem Teil der Gemeinwohlökonomie, verstanden als solidarische Ökonomie mit lokalem oder regionalem Bezug. Aus der Notwendigkeit, die Bürgerinnen und Bürger bei der Energiewende mitzunehmen, die vonseiten der politisch Verantwortlichen stets betont wird, können veränderte, den konventionell-kapitalistischen Produktionsprozessen alternativ gegenüberstehende Vorstellungen des Wirtschaftens resultieren, die eher auf Partizipationsförderung, ökologische und soziale Nachhaltigkeit statt Kapitalakkumulation ausgerichtet sind. Eine solche Prämissenverschiebung würde sich den oben skizzierten Vorstellungen eines materiellen Gegenmodells der solidarischen Ökonomie zum neoliberalen Wirtschaftssystem zumindest annähern. Gleiches gilt für die dezentrale Ausrichtung der Energieversorgungsstruktur, die in den Genossenschaften ihren Ausdruck findet.

Für die Ausschöpfung von Partizipationsbereitschaft und Dezentralität bietet sich die Genossenschaft als Organisationsform aufgrund der oben genannten genossenschaftlichen Prinzipien im besonderen Maße an. Flieger (2011, S. 58) geht sogar so weit zu sagen, die Genossenschaft sei „als privatrechtliches Unternehmen die einzig wirkliche Alternative zur gemeinwesenverantwortlichen staatlichen Organisation der Energieversorgung“.

\subsection{Energiegenossenschaften - eine demo- kratische und gemeinwesenorientierte Alternative der Energiewirtschaft}

Energiegenossenschaften, so unsere These, stellen also eine geeignete, vor allem aber eine demokratische Wirtschaftsund Organisationsform dar, deren positive Entwick- 
lung sich auch in den kommenden Jahren fortsetzen wird. Doch bedarf es zur Stabilisierung dieses Trends in jedem Fall eines höheren Grades an öffentlicher Publizität sowie empirischer Befunde, die die Potenziale und Entwicklungsmöglichkeiten einer genossenschaftlich organisierten Energiewirtschaft wissenschaftlich untermauern.

Erste Annäherungen bieten die von Volz (2011) bundesweit durchgeführte Vollerhebung von Energiegenossenschaften sowie die Befunde einer Studie des Klaus Novy Instituts (2012), in der im letzten Jahr sämtliche in Deutschland tätige Energiegenossenschaften erfasst und analysiert wurden. Die Erhebungen zeigen, dass die Ursachen der quantitativen Entwicklung der Energiegenossenschaften eher in den energiewirtschaftlichen Rahmenbedingungen (EEG und Einspeisevergütung) als in der Novellierung des Genossenschaftsgesetzes zu suchen sind. Energiegenossenschaften, deren Tätigkeitsschwerpunkte zumeist im Bereich der Photovoltaik liegen, sind in den letzten Jahren vor allem im ländlichen Raum entstanden. Entlang einer Typologie handelt es sich hierbei in erster Linie um Energie-Erzeuger-Genossenschaften, mit Abstand gefolgt von Energie-VerbraucherGenossenschaften, die auch regionale Netze betreiben. ${ }^{\circ}$

$\mathrm{Zu}$ beobachten ist eine Fülle unterschiedlicher Arrangements zwischen Energiegenossenschaften, Kommunen und Stadtwerken. Diese Arrangements zwischen sozialökologischem und kommunalwirtschaftlichem Umbaumodell der Energiewirtschaft (Mautz/Rosenbaum 2012, S. 87ff.) gilt es auszuleuchten, um die Potenziale von Energiegenossenschaften abzuschätzen.

Interessant hinsichtlich der Einordnung von Energiegenossenschaften in die Konzepte alternativer Wirtschaftsformen sind die Motive, die für die Genossenschaftsmitglieder bei der Gründung ausschlaggebend waren. Hier zeigt sich, dass die Motive zur Gründung der Genossenschaften relativ gleichrangig zwischen sozialen, wirtschaftlichen und energiepolitischen Anforderungen liegen (Klemisch 2012, S. 52). In jedem Fall handelt es sich in der Regel um Engagementaffine Genossenschaften (Alscher 2011, S.6ff.). Ein Befund, den es bei der Verortung der genossenschaftlich organisierten Energiewirtschaft innerhalb der Alternativformen zur kapitalistischen Wirtschaftsweise zu berücksichtigen gilt.

\section{Genossenschaften und ökonomische Reformansätze: erste Befunde, Forschungsfragen, gewerkschaftliche Perspektiven}

\subsection{Kann heute von einer Renaissance der Genossenschaften gesprochen werden?}

Die Erschließung neuer Geschäftsfelder, wie im Bereich der regenerativen Energieversorgung, scheint einer optimisti- schen Haltung hinsichtlich eines anhaltenden Auftriebes des Genossenschaftssektors auch in anderen Sektoren Recht zu geben. Nicht übersehen werden sollte bei aller Euphorie jedoch die Relation, in der die Rechtsform „eingetragene Genossenschaft" gegenüber anderen Unternehmensformen steht. Hier pflegt sie weiterhin ein Schattendasein. Hinsichtlich der quantitativen Entwicklung der Genossenschaften in den letzten Jahren bleibt unsere Prognose, gestützt auf die aktuelle Faktenlage, optimistisch, wenngleich eine Gleichrangigkeit mit der $\mathrm{GmbH}$ weder in der wirtschaftswissenschaftlichen Forschung und Lehre noch in der wirtschaftlichen Praxis zu erwarten ist.

Wie gezeigt wurde, bedarf es für eine fundierte Analyse von genossenschaftlichen Potenzialen einer genauen Differenzierung zwischen den jeweiligen Geschäftsfeldern, in denen Genossenschaften tätig sind. Befunde aus dem Gründungsgeschehen der Energiegenossenschaften weisen darauf hin, dass es vor allem darauf ankommt, präzise Geschäftsmodelle zu entwickeln, die als Blaupause gestreut werden und damit einen Nachahmungseffekt erzeugen.

\subsection{Ist mit der Entwicklung der Genossenschaf- ten eine Renaissance für gemeinschaftlich dezentrale Reformansätze verbunden?}

Eine gewisse Ambivalenz bleibt bei der Frage nach der neuen Blüte gemeinschaftlich dezentralen Wirtschaftens in Bezug auf Genossenschaften. Klar ist jedenfalls, dass sie immer sowohl Akteure des Marktes als auch der Zivilgesellschaft sind.

Der Forschung bleibt zu klären, welche Bedeutung den genossenschaftlichen Prinzipien und Förderzwecken beigemessen wird. Die Erkenntnis, dass nicht überall auch ideell Genossenschaft drin ist, wo formal eine Genossenschaft besteht, sollte in der Diskussion, wie die genossenschaftliche Organisationsform innerhalb alternativer Wirtschaftsformen wie der Solidarischen Ökonomie und Gemeinwohlökonomie zu verorten ist, Berücksichtigung finden. Entlang der genossenschaftlichen Werte wie Solidarität und Gemeinsinn muss daher im Einzelfall geprüft werden, inwieweit die in diesen Konzeptionen maßgeblichen Werte gelebt und gefördert werden und somit gemeinschaftlichen, dezentralen Reformansätzen entsprechen.

Ob die neu gründeten Energiegenossenschaften den beschriebenen Genossenschaftsgeist verwirklichen, lässt sich zum heutigen Zeitpunkt nicht abschließend beurteilen. Die empirischen Ergebnisse der oben aufgeführten Studien (Volz 2011; Klaus Novy Institut 2012) geben jedoch erste Hinweise, dass sich die Gründungsmotive relativ gleichge-

6 Die in Deutschland wohl bekannteste Erneuerbare-EnergieGenossenschaft Greenpeace Energy eG ist als überregionaler Stromanbieter in der Struktur der Erneuerbare-Energie-Genossenschaften eher eine Ausnahme. 
wichtig an den Kriterien der Nachhaltigkeit (Ökonomie, Ökologie und Soziales) orientieren.

Sich dem Gegenstandsbereich der Neugründungen und der Entwicklung von genossenschaftsadäquaten Geschäftsfeldern und situationsgebundenen Einsatzmöglichkeiten in systematischer empirischer Form zu nähern, wäre daher nützlich und wünschenswert. Die traditionelle Genossenschaftssystematik, die bisher der Analyse des Gründungsgeschehens zugrunde gelegt wird, ist hierbei weniger zielführend als z. B. ein Branchenzugang, der auf jeden Fall ergänzt werden sollte.

Neben eher praktischen Fragen, wie z. B. dem sozialökologischen Modell des energiewirtschaftlichen Umbaus mittels Genossenschaftsgründungen zum Durchbruch verholfen werden kann, halten wir es für wichtig, die Fragestellung von Geschäftsmodellen für Genossenschaften in sozialen, ökologischen und kulturellen Aufgabengebieten zu schärfen und mit wissenschaftlichem Sachverstand zu hinterlegen.

\subsection{Können Genossenschaften überhaupt als Teil der wirtschaftlichen Reformansätze verstanden werden?}

Auch diese Frage sperrt sich gegen allzu klare Antworten. Die beschriebenen Genossenschaftssektoren sind sicherlich mehrheitlich dem ersten Sektor einer privatkapitalistischen Wirtschaft zuzurechnen. Gleichwohl stecken in jeder Genossenschaft Strukturen, Werte und Funktionsweisen, die den Anforderungen einer solidarischen Ökonomie entsprechen und somit einen sozialreformerischen Charakter aufweisen.

Eine Verknüpfung von Theorie- und Praxisanbindung der Genossenschaftsthematik und Genossenschaftsforschung an Konzepte einer moralischen Ökonomie und nicht nur die Übertragung der gängigen Managementlehre auf die Genossenschaften wäre wünschenswert, um eine wirtschafts- und sozialwissenschaftliche Debatte um den sozialreformerischen Status von Genossenschaften zu führen sowie eine Wirtschaftsreformpraxis neu anzuschieben (Novy 1979). Fernziel wäre neben der Förderung des Genossenschaftsgedankens die Etablierung eines neuen Diskurses um Fragen der Wirtschaftsdemokratie, der Genossenschaften im Spannungsfeld von Erstem und Dritten Sektor thematisiert. Hierdurch könnte u. a. die enge Fixierung auf eine einzelbetriebliche und tarifpolitische Mitbestimmungsdiskussion, wie sie in den Gewerkschaften mit Vorrang geführt werden, aufgebrochen werden. Die Gewerkschaften sollten sich an diesem Diskurs genauso beteiligen wie an der Förderung des Genossenschaftsgedankens. Betriebsübernahmen durch Belegschaften und Unternehmensnachfolgen sind hier potenzielle Handlungsfelder für genossenschaftliche Lösungen.

\section{LITERATUR}

Alscher, M. (2011): Genossenschaften - Akteure des Marktes und der Zivilgesellschaft, Berlin

Altvater, E. (2012): Genossenschaft und gutes Leben - Der Sozialismus des 21. Jahrhunderts, in: Blätter für deutsche und internationale Politik (4), S. 53-63 Altvater, E./Sekler, N. (Hrsg.) (2006): Solidarische Ökonomie. Reader des Wissenschaftlichen Beirats von Attac, Hamburg

Asendorf, I./Demmeler, M./Flieger, B./Jaudas,J./Sauer, D./Scholz, S. (2003): Nachhaltigkeit durch regionale Vernetzung - Erzeuger-Verbraucher-Gemeinschaften im Bedürfnisfeld Ernährung, Endbericht zum gleichnamigen Forschungsprojekt, Dorfen

Atmaca, D. (2007): Produktivgenossenschaften - zwischen Utopie und Realismus, in: Brockmeier, T./Fehl, U. (Hrsg.): VolkswirtschaftlicheTheorie der Kooperation in Genossenschaften, S. 509-590

Bierbaum, H./Riege, M. (Hrsg.) (1985): Die neue Genossenschaftsbewegung, Hamburg

Bierbaum, H./Riege, M. (1989): Selbsthilfe, Genossenschaften, Vergesellschaftung, Hamburg

Bispinck, R./Schulten T./Raane P. (Hrsg.) (2008): Wirtschaftsdemokratie und expansive Lohnpolitik - Zur Aktualität von Viktor Agartz, Hamburg

Blome-Drees J. (2010): Ansatzpunkte zu einer Erhöhung der Neugründungsquote von Genossenschaften, in: Münkner H.-H./Ringle G. (Hrsg.): Neue Genossenschaften und innovative Aktionsfelder - Grundlagen und Fallstudien, Baden-Baden, S. 23-36

Bontrup, H.-.J./Marquardt, R.-M. (2010): Kritisches Handbuch der deutschen Elektrizitätswirtschaft, Berlin

Brinkmann, V. (2010): Sozialwirtschaft: Grundlagen - Modelle - Finanzierung, Wiesbaden

Bundesministerium für Umwelt, Naturschutz und Reaktorsicherheit (BMU)

(Hrsg.) (2011): Erneuerbare Energien. Innovationen für eine nachhaltige Energiezukunft, Berlin

Demirovic A. (2007): Demokratie in der Wirtschaft. Positionen - Probleme Perspektiven, Münster

Demirovic A. (2011): Wirtschaftsdemokratie nach ihrem Scheitern, in: Friedrich Ebert-Stiftung (FES) (Hrsg.): Zukunft der Demokratie - Demokratie der Zukunft, Hannover, S. 46-65

Elsen, S. (1998): Gemeinwesenarbeit und Gemeinwesenökonomie im Zeitalter der Globalisierung, in: Klöck, T. (Hrsg.): Solidarische Ökonomie und Empowerment, Neu-Ulm, S. 69-98

Elsen, S. (Hrsg.) (2011): Ökosoziale Transformation - Solidarische Ökonomie und die Gestaltung des Gemeinwesens, Neu-Ulm

Elsen, S. (2011): Solidarische Ökonomie, die Wiederentdeckung der Commons und ökosoziale Entwicklung, in: Elsen, S. (Hrsg.) : Ökosoziale Transformation Solidarische Ökonomie und die Gestaltung des Gemeinwesens, Neu-Ulm, S. $90-114$

Engelhardt, W. (1985): Allgemeine Ideengeschichte des Genossenschaftswesens. Einführung in die Genossenschafts- und Kooperationslehre auf geschichtlicher Basis, Darmstadt

Eschenburg, R. (1971): Ökonomische Theorie der genossenschaftlichen Zusammenarbeit, Tübingen

Felber, C. (2010): Gemeinwohlökonomie - Das Wirtschaftsmodell der Zukunft, Wien

Flieger, B. (2006): Genossenschaften in Deutschland - Teil der Solidarischen Ökonomie?, in: Altvater, E./Sekler, N. (Hrsg.) (2006): Solidarische Ökonomie. Reader des Wissenschaftlichen Beirats von Attac, Hamburg, S. 47-61

Flieger, B. (2011): Wirtschaftliche Beteiligung im kommunalen Klimaschutz Energiegenossenschaften: Bürgerbeteiligung an der kommunal organisierten Energiewende, in: Heinrich-Böll-Stiftung Brandenburg (Hrsg.): Bürgerbeteiligung im kommunalen Klimaschutz. Antworten europäischer Städte und Gemeinden, Potsdam

Flieger B./Klemisch H. (2008): Eine andere Energiewirtschaft ist möglich, Neue Energiegenossenschaften, in: Widerspruch 28 (54), S. 105-110

GdW-Bundesverband deutscher Wohnungs- und Immobilienunternehmen e.V. (Hrsg.) (2012): GdW kompakt Teil 1: Internationales Jahr der Genossenschaften, Berlin

Geschwandtner, M./Helios, M. (2006): Genossenschaftsrecht - das neue Genossenschaftsgesetz und die Einführung der europäischen Genossenschaft, Freiburg 
Giegold, S./Embshoff, D. (2008): Solidarische Ökonomie im globalisierten Kapitalismus, in: Giegold, S./Embshoff, D. (Hrsg.): Solidarische Ökonomie im globalisierten Kapitalismus, in Kooperation mit der „Bewegungsakademie“ und der "tageszeitung," Hamburg, S. 11-24

Giegold, S./Embshoff, D. (Hrsg.) (2008): Solidarische Ökonomie im globalisier ten Kapitalismus, in Kooperation mit der "Bewegungsakademie" und der "tageszeitung", Hamburg

Göler von Ravensburg, N. (2011): Genossenschaftliche Neugründungen - Unterstützung durch die Wissenschaft, in: Zeitschrift für das gesamte Genossenschaftswesen (ZfgG) 61 (2), S. 127-140

Gräber, D. (2012): Inside Occupy, Frankfurt a. M.

Grosskopf, W./Münkner, H.-H./Ringle, G. (2012): Unsere Genossenschaft, Wies baden

Hettlage, R. (1990): "Solidarität" und "Kooperationsgeist" in genossenschaftlichen Unternehmungen, in: Arbeitskreis für Kooperation und Partizipation (Hrsg.): Kooperatives Management, Baden-Baden, S. 123-152

innova - Verein zur Förderung des Genossenschaftsgedankens e.V. (Hrsg.) (1990): Perspektiven der Genossenschaften - Impulse für eine demokratische Marktwirtschaft, Darmstadt

Jenkis, H. W. (1992): Wohnungsbaugenossenschaft in Deutschland, in: Mändle, E./Swoboda, W. (Hrsg.): Genossenschaftslexikon, Wiesbaden, S. $733-735$

Kerber-Clasen, S. (2012): Produktivgenossenschaften und solidarische Ökonomie als Forschungs- und Praxisfeld: Eine Skizze, in: WSI-Mitteilungen 65 (4), S. 281-288, http://www.boeckler.de/wsimit_2012_04_kerber.pdf

Klaus Novy Institut (2012): Genossenschaftliche Unterstützungsstrukturen für eine sozialräumlich orientierte Energiewirtschaft, Köln

Klemisch, H. (2012): Energiegenossenschaften - Rekommunalisierung der Energiewirtschaft, in: Alternative Kommunalpolitik (akp) (4), S.48-53

Klemisch, H./Flieger, B. (2007): Genossenschaften und ihre Potenziale für Innovation, Partizipation und Beschäftigung. Der Beitrag von Genossenschaften zur sozialen Verantwortung von Unternehmen, hrsg. vom Klaus-Novy-Institut, KNi BERICHT (01), Köln

Klemisch, H./Maron, H. (2010): Genossenschaftliche Lösungsansätze zur Sicherung der kommunalen Daseinsvorsorge, in: Zeitschrift für das gesamte Genossenschaftswesen (ZfgG) 60 (1), S. 3-13

Koch, H. (2007): Soziale Kapitalisten, Berlin

Laurinkari, J./Brazda, J. (1990): Genossenschaftliche Grundwerte, in: Laurinkari, J./Brazda, J. (Hrsg.): Genossenschaftswesen. Hand- und Lehrbuch,

München, S. 70-77

Mändle, E. (2005): Grundriss der Wohnungsgenossenschaften, Hamburg

Martens, H. (2010): Neue Wirtschaftsdemokratie. Anknüpfungspunkte im Zeichen der Krise von Ökonomie, Ökologie und Politik, Hamburg

Mautz, R./Rosenbaum, W. (2012): Der deutschen Stromsektor im Spannungsfeld energiewirtschaftlicher Umbaumodelle, in: WSI-Mitteilungen 65 (2),

S. 85-93, http://www.boeckler.de/wsimit_2012_02_mautz.pdf

Mersmann, A./Novy, K. (1991): Gewerkschaften, Genossenschaften, Gemeinwirtschaft. Hat eine Ökonomie der Solidarität eine Chance?, Köln

Müller, M./Schaltegger, S. (Hrsg.) (2008): Corporate social responsibility Trend oder Modeerscheinung, München

Müller-Plantenberg, C. (2007): Solidarische Ökonomie und Regionale Entwicklung, in: Müller-Plantenberg, C. (Hrsg.): Solidarische Ökonomie in Europa. Betriebe und regionale Entwicklung/Internationale Sommerschule in Imshausen, Kassel, S. 55-67

Müller-Plantenberg, C./Nitsch, W./Schlosser, I./Loccumer Initiative (Hrsg.) (2007): Solidarische Ökonomie in Europa. Betriebe und regionale Entwicklung/ Internationale Sommerschule in Imshausen, Kassel

Münkner H.-H./Ringle G. (Hrsg.) (2010): Neue Genossenschaften und innovative Aktionsfelder- Grundlagen und Fallstudien, Baden-Baden

Nida-Rümelin, J. (2012): Die Optimierungsfalle - Philosophie einer humanen Ökonomie, München
Novy, K. (1979): Wirtschaftswissenschaften und Wirtschaftsreformpraxis, in: Leviathan 7 (4), S.466-490

Novy, K. (1982): Vorwärts oder rückwärts?, in: Benseler, F./Heinze, R G./KIönne,

A. (Hrsg.): Zukunft der Arbeit, Hamburg, S.119-130

Novy, K. (1985): Vorwärts immer - rückwärts nimmer, in: Bierbaum H./Riege,

M. (Hrsg.): Die neue Genossenschaftsbewegung Hamburg, S. 124-141

Novy , K. (1990): Vergenossenschaftlichung Osteuropas?, in: innova (Hrsg.)

a.a.O., S. 7-15

Notz, G. (2011): Theorien alternativen Wirtschaftens - Fenster in eine andere Welt, Stuttgart

Scheer H. (2010): Der energetische Imperativ, München

Schweizer-Ries, P. (2010): Aktivität und Teilhabe - Akzeptanz Erneuerbarer Energien durch Beteiligung steigern, Magdeburg

Sedlacek, T. (2012): Die Ökonomie von Gut und Böse, München

Stappel, M. (2006): Die deutschen Genossenschaften 2006. Entwicklungen Meinungen - Zahlen, Wiesbaden

Stappel M. (2011): Genossenschaften in Deutschland, Frankfurt a. M

Theurl T./Wendler C. (2011): Was weiß Deutschland über Genossenschaften? Aachen

trend:research (2011): Marktakteure Erneuerbare-Energie-Anlagen in der Stromerzeugung, www.kni.de

Verein zur Förderung der solidarischen Ökonomie e.V. (Hrsg.) (2011): Schritte auf dem Weg zur solidarischen Ökonomie, Kassel

Vogt, W. (2011): Genossenschaften - eine andere Form des Wirtschaften, Berlin Volz, R. (2010): Stand und Entwicklungsmöglichkeiten von Bürgerenergiegenossenschaften in Deutschland, Hohenheim

Volz, R. (2011): Strukturen und Merkmale von Energiegenossenschaften in Deutschland - Ausgewählte Ergebnisse einer bundesweit durchgeführten Vollerhebung, in: Doluschitz, R. et al. (Hrsg.): Hohenheimer Genossenschaftsforschung, Stuttgart

Voß, E. (2010): Wegweiser Solidarische Ökonomie. Anders wirtschaften ist möglich!, Neu-UIm

Winter, H.-W./Mändle, E. (1980): Genossenschaftliches Demokratieprinzip, in: Mändle, E./Winter, H.-W. (Hrsg.): Handwörterbuch des Genossenschaftswesens, Spalte 253-261, Wiesbaden

\section{AUTOREN}

HERBERT KLEMISCH, Dr., Wirtschafts- und Sozialwissenschaftler, ist Projektmitarbeiter im Wissenschaftsladen Bonn und war bis vor Kurzem Projektleiter im Klaus Novy Institut Köln. Arbeitsschwerpunkte: Umwelt-, Arbeits-, Partizipations- und Genossenschaftsforschung, Evaluation von Ökologie- und Nachhaltigkeitsprozessen.

\section{hk@umwelt-evaluation.de}

MORITZ BODDENBERG, studiert Soziologie im Masterstudiengang der Universität Frankfurt und war studentischer Mitarbeiter im Klaus Novy Institut. Arbeitsschwerpunkt: Solidarität in der globalisierten Ökonomie.

moritz-boddenberg@web.de 